\title{
Evidências científicas brasileiras acerca da infecção primária da corrente sanguínea em pediatria*
}

\section{Brazilian scientific evidences about primary bloodstream infection in pediatrics}

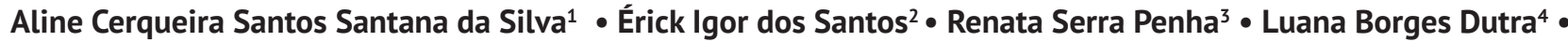 \\ Raphael Neves Barreiros ${ }^{5} \bullet$ Isabela Valente Ribeiro ${ }^{6}$
}

\section{RESUMO}

Objetiva-se analisar as evidências científicas brasileiras mais recentes sobre prevenção e controle da Infecção Primária de Corrente Sanguínea (IPCS) em pediatria. Tratou-se de uma revisão integrativa de literatura de artigos publicados nos últimos dez anos na base de dados Literatura Latino-Americana do Caribe em Ciências da Saúde (LILACS) e nas bibliotecas virtuais Biblioteca Virtual em Saúde (BVS) e Scientific Eletronic Library Online (SciELO). Foram combinados os descritores em português "Cateteres" AND "Infecções relacionadas a cateter" AND "Infecção Hospitalar AND Pediatria". Foram selecionados 11 artigos após aplicação dos critérios de inclusão e exclusão. As evidências apontam para a prevalência de IPCS, contudo, estas podem ser prevenidas quando ocorre a adoção de boas práticas pelos serviços de saúde. Concluí-se que a pesar das medidas de prevenção e controle da IPCS estarem bem estabelecidas, as evidências apontam níveis de desempenho insatisfatórios pelos profissionais de saúde na implementação das mesmas.

Palavras-chave: Enfermagem Pediátrica; Infecção Hospitalar; Cateteres.

\begin{abstract}
The aim is to analyze the latest Brazilian scientific evidence on control and prevention of Primary Bloodstream Infection (IPCS) in pediatrics. Integrative literature review of articles published in the last ten years in the Latin American Database Caribbean Health Sciences (LILACS) and virtual libraries Virtual Health Library (VHL) and Scientific Electronic Library Online (SciELO). The descriptors were combined in Portuguese "Catheters" AND "infections related to catheter" AND "Hospital Infection AND Pediatrics". 11 articles were selected after applying the inclusion and exclusion criteria's. Evidence points to the prevalence of IPCS; however, these can be prevented when you apply best practices for health services. It concludes that despite the prevention measures and control of IPCS being well established, evidence shows unsatisfactory performance levels by the health professionals in the implementation.
\end{abstract}

Keywords: Pediatric Nursing, Cross Infection, Catheters.

${ }^{1}$ Enfermeira, Doutora em Enfermagem pela Universidade Federal do Rio de Janeiro, Professora Assistente da Universidade Federal Fluminense, Campus Rio das Ostras/RJ. E-mail: alinecer2014@gmail.com

${ }^{2}$ Enfermeiro, Doutor em Enfermagem pelo Programa de Pós-Graduação em Enfermagem da UERJ, Professor Assistente da Universidade Federal Fluminense, Campus Rio das Ostras/RJ.E-mail: eigoruff@gmail.com

${ }^{3}$ Enfermeira, Graduada em Enfermagem pela Universidade Federal Fluminense, Campus Rio das Ostras/RJ. E-mail: renataspenha@hotmail.com

${ }^{4}$ Enfermeira, Graduada em Enfermagem pela Universidade Federal Fluminense, Campus Rio das Ostras/RJ. E-mail: luanaborges@id.uff.br

${ }^{5}$ Enfermeiro, Graduado em Enfermagem pela Universidade Federal Fluminense, Campus Rio das Ostras/RJ. E-mail: raphaelbarreriros@id.uff.br

${ }^{6}$ Enfermeira, Graduada em Enfermagem pela Universidade Federal Fluminense, Campus Rio das Ostras/RJ. E-mail: isabelavalenteribeiro@gmail.com 


\section{INTRODUÇÃO}

No século XX, menos de $20 \%$ dos pacientes que se encontravam hospitalizados recebiam o tratamento através da terapia intravenosa (TI). Nos dias atuais essa estimativa chega a atingir $90 \%$, tornando-se um dos procedimentos invasivos mais realizados na rotina pelos profissionais de enfermagem ${ }^{1}$. Todavia, esta medida torna o paciente cada vez mais suscetível à eventos adversos infecciosos e não infecciosos ${ }^{2}$.

Tal fato, explica-se através da crescente utilização da TI como forma mais comum de intervenção no cuidado à saúde $^{3}$, onde aproximadamente 1 a $10 \%$ dos pacientes que são submetidos à mesma desenvolvem infecções locais ou sistêmicas, devido alteração da pele como barreira, possibilitando a translocação de microrganismos à via hematogênica ${ }^{2}$

Sobre este aspecto, verifica-se na literatura atual a frequente ocorrência de erros e riscos ocasionados aos pacientes submetidos à mesma, especialmente nas áreas destinadas ao atendimento neonatal e pediátrico, concorrendo em grande parte ao aumento da morbidade, da mortalidade e dos custos decorrentes do tratamento instituído ${ }^{3}$.

Com o grande advento tecnológico direcionado ao aprimoramento de dispositivos intravasculares, surgem materiais cada vez mais específicos para o atendimento de pacientes em estado crítico, o que os torna indispensáveis à assistência à saúde ${ }^{4}$

A terapia intravenosa, por ser a mais utilizada, compreende um conjunto de conhecimentos e técnicas que incluem desde a inserção do cateter até os cuidados que englobam a manutenção, salinização, troca de cobertura e descarte do dispositivo, perpassando a administração de soluções e medicamentos no sistema circulatório ${ }^{5-6}$.

Nesta leitura, a Infecção Primária da Corrente Sanguínea (IPCS) associada à inserção e manutenção de cateteres venosos centrais em neonatos e crianças apresenta-se como uma das complicações mais comuns apresentadas pelo paciente no ambiente hospitalar, resultando em maior tempo de permanência no hospital ${ }^{7}$. A IPCS é aquela infecção de consequências sistêmicas graves, ocasionando bacteremia ou sepse, sem foco primário identificável, o que torna difícil determinar o envolvimento do cateter venoso central (CVC) em vigência da mesma ${ }^{8-9}$.

Assim, as infecções relacionadas à assistência à saúde (IRAS) se apresentam, na atualidade, como a complicação mais frequente, com notório destaque à IPCS, sendo o CVC e sua manipulação a principal causa ${ }^{10}$

Esse evento ganha intensidade no contexto da unidade de terapia intensiva (UTI), devido maior tempo de permanência do paciente neste setor e também pela manipulação excessiva do dispositivo intravenoso, o que potencializa a colonização da corrente sanguínea ${ }^{8-10}$

No Brasil, a sepse associada ao CVC representa um sério problema de saúde pública, que necessita da adoção de medidas de vigilância eficazes para reduzir os índices de infecções nosocomiais ${ }^{8-9}$

Mediante ao exposto, tendo como base os achados da literatura, este trabalho analisou as evidências científicas brasileiras mais recentes sobre prevenção e controle da Infecção Primária de Corrente Sanguínea (IPCS) em pediatria.

\section{MÉTODO}

Trata-se de um estudo descritivo, pautado na revisão integrativa de literatura, que visa à análise de pesquisas relevantes, sintetiza o conhecimento sobre determinada temática e identifica lacunas que necessitam ser preenchidas sobre um tema definido e específico ${ }^{11}$

Assim, foram percorridas as seguintes etapas, a saber. A primeira etapa foi composta pela identificação do problema com a definição da questão de pesquisa: quais as evidências cientificas brasileiras mais recentes sobre prevenção e controle da Infecção Primária de Corrente Sanguínea (IPCS) em pediatria?

A segunda etapa do estudo consistiu na busca de artigos nas bibliotecas virtuais ou bases de dados Biblioteca Virtual em Saúde (BVS), Scientific Eletronic Library Online (SciELO), Literatura Latino Americana do Caribe em Ciências da Saúde (LILACS), Literatura Internacional em Ciências da Saúde (MEDLINE), Base de Dados em Enfermagem (BDENF) e Portal de Periódicos da Capes. Delimitou-se, como recorte temporal, os últimos 10 anos, com o propósito de evidenciar as publicações mais recentes sobre a temática em questão. A busca dos artigos deu-se através dos descritores selecionados, sendo norteada através dos seguintes critérios de inclusão: artigos em português; publicados nos últimos dez anos; que abordassem pacientes pediátricos e recém-nascidos, disponíveis gratuitamente em texto completo e que respondessem à pergunta de pesquisa.

Foram excluídos artigos que apresentassem duplicidade (esses foram contabilizados como apenas um), cujo link para acesso em texto completo apresentasse erro mediante a tentativa de acesso e artigos in press ou ahead of print, o que inviabilizaria o seu acesso em formato final. A busca dos artigos foi realizada por dois revisores. A coleta de dados ocorreu em janeiro de 2016. O levantamento realizado ocorreu através dos descritores em saúde disponíveis no Portal de Descritores em Ciências da Saúde (DeCS). A seleção dos descritores foi norteada por sua proximidade ao objeto em questão. Chegou-se a seguinte combinação: "Cateteres" AND "Infecções relacionadas a cateter" AND "Infecção Hospitalar" AND "Pediatria”, em inglês, português e espanhol.

Por seu turno a terceira etapa consistiu no lançamento dos estudos em um instrumento de coleta de dados confeccionado pelos autores, que contou com as seguintes variáveis: nome dos autores, ano de publicação, periódico, base de dados, título do artigo, síntese do conhecimento e nível de evidência. 
A seguir foi realizada a quarta etapa composta pela tabulação dos estudos no software Microsoft Excel 2010 para organização e sumarização das principais informações, constituindo um banco de dados. Foi realizada uma avaliação individual de cada estudo para uma análise crítica através de leituras do texto completo como forma de identificar sua aderência ou não ao objetivo desta pesquisa ${ }^{11}$. Após a definição do número final de artigos para compor a revisão, são realizadas a quinta e sexta etapas, que dizem respeito ao agrupamento dos resultados (evidências) em categorias internamente homogêneas e heterogêneas entre si, para, posteriormente, ser apresentada a síntese do conhecimento.

\section{RESULTADOS}

\section{Caracterização quantitativa das publicações}

Foram encontrados 810 artigos após aplicação dos critérios de inclusão e de exclusão previamente estabelecidos, foram selecionados 11 artigos, como verifica-se na figura 1. Dos 11 artigos, sete foram encontrados na LILACS, um na MEDLINE e três na BDENF.

Os artigos selecionados foram publicados entre os anos 2005 a 2015. Houve uma publicação nos anos de 2009, 2011 e 2014, duas publicações nos anos de 2010 e 2012 e quatro publicações no ano de 2013, o que se pode inferir um crescimento na busca de novos conhecimentos acerca da adoção de boas práticas para o controle da infecção primária da corrente sanguínea em pediatria.

Considerando o local de publicação, a região sudeste destaca-se como a maior produtora de conhecimentos nesta temática abrangendo cerca de nove artigos das produções, seguida da região Sul e centro-oeste com um artigo da produção, cada.

É importante ressaltar, que a categoria profissional com maior número de publicações nesta vertente foram os enfermeiros, abarcando dez produções, seguido por médicos e farmacêuticos, envolvidos em um único artigo, cada.

Após leitura e aproximação dos estudos foram evidenciadas duas categorias quais sejam, 'Adoção de boas práticas pelos serviços de saúde como estratégia para o controle da IPCS' e 'A sepse neonatal e pediátrica como principal complicação relacionada à IPCS'.

As evidências dos artigos foram avaliadas por meio do sistema de classificação hierárquica da qualidade das evidências. A cada artigo foi atribuído um conceito que variou entre 1 a 6 conforme sua força de evidência ${ }^{12}$.

Em seguida será apresentado o quadro 1 (dividido em duas partes) referente aos dados dos artigos identificados durante a Revisão Integrativa de Literatura (RIL).

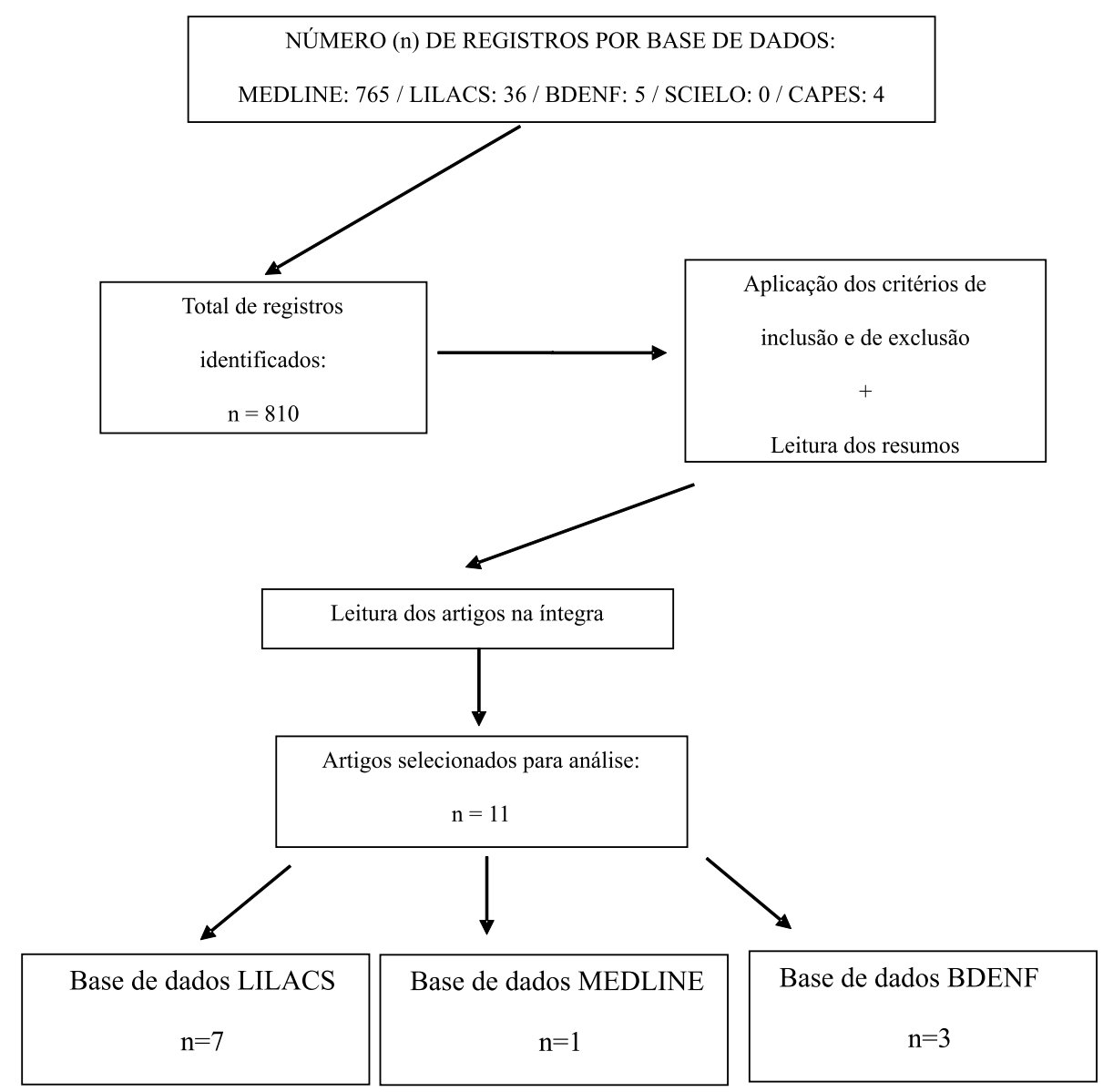

Figura 1: Fluxograma das etapas metodológicas cumpridas para a seleção dos artigos - Rio de Janeiro - RJ, Brasil. Fonte: dados da pesquisa. 
Quadro 1 - Artigos acerca da infecção primária da corrente sanguínea em pediatria de 2005 a 2015, identificados após RIL. Rio de Janeiro, RJ, Brasil, 2015. (Parte 1)

\begin{tabular}{|c|c|c|c|c|c|}
\hline Autor & Ano & $\begin{array}{c}\text { Periódico/ Base } \\
\text { de dados }\end{array}$ & Título do artigo & Síntese dos Resultados & $\begin{array}{l}\text { Nível de } \\
\text { Evidência }\end{array}$ \\
\hline $\begin{array}{l}\text { Corrêa KLG; } \\
\text { Almeida GMD; } \\
\text { Almeida JJN; } \\
\text { Rossi F4 }\end{array}$ & 2012 & $\begin{array}{l}\text { Jornal Brasileiro } \\
\text { de Patologia } \\
\text { e Medicina } \\
\text { Laboratorial/ } \\
\text { LILACS }\end{array}$ & $\begin{array}{l}\text { Diferença de tempo de } \\
\text { positividade: método útil no } \\
\text { diagnóstico de infecção da } \\
\text { corrente sanguínea } \\
\text { relacionada com cateter? }\end{array}$ & $\begin{array}{l}\text { Utilização da DTP como ferramenta } \\
\text { auxiliar no diagnóstico das infecções } \\
\text { de corrente sanguínea relacionadas } \\
\text { com cateter (ICSRC) desde que a coleta } \\
\text { seja rigorosamente padronizada. }\end{array}$ & 4 \\
\hline $\begin{array}{l}\text { Moncaio ACS; } \\
\text { Figueiredo RM }{ }^{6}\end{array}$ & 2009 & $\begin{array}{l}\text { Revista Eletrônica } \\
\text { de Enfermagem/ } \\
\text { BDENF }\end{array}$ & $\begin{array}{l}\text { Conhecimento e práticas no } \\
\text { uso do cateter periférico pela } \\
\text { equipe de enfermagem }\end{array}$ & $\begin{array}{l}\text { Evidencia que apesar do conhecimento } \\
\text { teórico da equipe de enfermagem } \\
\text { ter sido adequado, o mesmo não foi } \\
\text { implementado na prática, denotando } \\
\text { a necessidade de estabelecer práticas } \\
\text { educativas e administrativas eficazes } \\
\text { como medidas de prevenção e controle. }\end{array}$ & 6 \\
\hline $\begin{array}{l}\text { Vilela R; } \\
\text { Dantas SRPE; } \\
\text { Trabasso } \mathrm{P}^{7}\end{array}$ & 2010 & $\begin{array}{l}\text { Revista Paulista } \\
\text { de Pediatria / } \\
\text { LILACS }\end{array}$ & $\begin{array}{l}\text { Equipe interdisciplinar reduz } \\
\text { infecção sanguínea relacionada } \\
\text { ao cateter venoso central em } \\
\text { Unidade de Terapia Intensiva } \\
\text { Pediátrica }\end{array}$ & $\begin{array}{l}\text { O estudo avalia duas formas de } \\
\text { medidas de prevenção: medida } \\
\text { de intervenção indireta de caráter } \\
\text { educativo e medidas de intervenção } \\
\text { direta. }\end{array}$ & 4 \\
\hline $\begin{array}{l}\text { Jardim JM; } \\
\text { Lacerda RA; } \\
\text { Soares NJD; } \\
\text { Nunes } \text { BK }^{8}\end{array}$ & 2013 & $\begin{array}{l}\text { Revista da Escola } \\
\text { de Enfermagem } \\
\text { da USP / } \\
\text { MEDLINE }\end{array}$ & $\begin{array}{l}\text { Avaliação das práticas } \\
\text { de prevenção e controle } \\
\text { de infecção da corrente } \\
\text { sanguínea em um hospital } \\
\text { governamental. }\end{array}$ & $\begin{array}{l}\text { Avaliação das práticas no controle e } \\
\text { prevenção das infecções da corrente } \\
\text { sanguínea, indicam a necessidade de } \\
\text { criação de estratégias educativas. }\end{array}$ & 4 \\
\hline $\begin{array}{l}\text { Cali K; Silvino } \\
\text { ZR; Valente } \\
\text { GSC }^{14}\end{array}$ & 2013 & $\begin{array}{l}\text { Online Brazilian } \\
\text { Journal of } \\
\text { Nursing / LILACS }\end{array}$ & $\begin{array}{l}\text { Bundle para manuseio do } \\
\text { Cateter Venoso Central: } \\
\text { pesquisa exploratória e } \\
\text { descritiva }\end{array}$ & $\begin{array}{l}\text { Orienta a adoção de medidas seguras } \\
\text { com o uso do bundle como uma } \\
\text { estratégia de intervenção de medida } \\
\text { simples e eficaz. }\end{array}$ & 6 \\
\hline $\begin{array}{l}\text { Prates DB; } \\
\text { Vieira MFM; } \\
\text { Leite TS; Couto } \\
\text { BRGM; Silva } \\
\text { EU }\end{array}$ & 2014 & $\begin{array}{c}\text { Revista médica } \\
\text { de Minas Gerais / } \\
\text { LILACS }\end{array}$ & $\begin{array}{l}\text { Impacto de programa } \\
\text { multidisciplinar para redução } \\
\text { de incidência de infecção } \\
\text { associada à assistência na UTI } \\
\text { de hospital terciário em Belo } \\
\text { Horizonte }\end{array}$ & $\begin{array}{l}\text { Mostrou algumas medidas de } \\
\text { intervenção na UTI de um hospital } \\
\text { terciário, através da equipe } \\
\text { multidisciplinar minimizando assim a } \\
\text { incidência e infecções e a melhoria na } \\
\text { assistência de saúde. }\end{array}$ & 4 \\
\hline
\end{tabular}

Fonte: dados da pesquisa.

\section{Categoria 1- A adoção de boas práticas pelos serviços de saúde como estratégia para o controle da IPCS}

Medidas de prevenção e controle da infecção da corrente sanguínea associada ao cateter venoso central são conhecidas, principalmente por meio de diretrizes de recomendações internacionais ${ }^{8-10}$ contudo, o maior de seus desafios na atualidade centra-se em reconhecer se as mesmas são, de fato, seguidas ${ }^{8}$

Uma pesquisa relatou que a IPCS, na atualidade, configura-se como um dos principais problemas de assistência à saúde, principalmente aqueles pacientes que necessitam para sua recuperação clínica da instalação e manutenção de um $\mathrm{CVC}^{9}$.

Essa afirmativa torna-se bastante evidente quando outros estudos corroboram esta assertiva ${ }^{13-14}$ ao analisarem dados internacionais e averiguarem que $48 \%$ dos pacientes em UTI portam um CVC, somando 15 milhões de cateteres por ano e que a incidência é de 5,3 infecções relacionadas ao cateter para cada 1000 cateteres por dia, ocasionando o aumento da morbidade e da mortalidade, chegando a $18 \%$ correspondendo a 14 mil óbitos por ano
Acrescido ao fato da grande densidade de incidência de pacientes de UTI portarem um CVC, chamam a atenção as complicações relacionadas a essas infecções, correspondendo no alto índice de mortalidade que varia entre 12 a 30\%-15, além dos custos adicionais que provocam aos sistemas de saúdes, que variam entre U\$ 60 a 460 milhões por ano, visto que esses pacientes, além de prolongarem sua permanência no âmbito hospitalar, necessitam de intervenções adicionais que aumentam a sua estadia em torno de 10 a 40 dias $^{4-16}$

Cabe destacar que, além dos riscos e complicações, a taxa de infecção apresentada por esta clientela torna-se muito difícil de ser estimada em virtude de fatores como a premente necessidade de procedimentos invasivos para instalação do CVC, sonda vesical, ventilação mecânica ${ }^{17} \mathrm{e}$ medidas adicionais adotadas face a esses procedimento ${ }^{4-18}$

Em se tratando da população neonatal e pediátrica admitidas em UTI esta situação se complexifica à medida em que os afetam a lenta maturação do seu sistema imunológico (cujo desenvolvimento é menos acentuado quanto menor for a idade, tornando maior o risco de aquisição de doenças transmissíveis), o compartilhamento 
Quadro 2 - Artigos acerca da infecção primária da corrente sanguínea em pediatria de 2005 a 2015, identificados após RIL. Rio de Janeiro, RJ, Brasil, 2015. (Parte 2)

\begin{tabular}{|c|c|c|c|c|c|}
\hline Autor & Ano & $\begin{array}{c}\text { Periódico/ Base } \\
\text { de dados }\end{array}$ & Título do artigo & Síntese dos Resultados & $\begin{array}{c}\text { Nível de } \\
\text { Evidência }\end{array}$ \\
\hline $\begin{array}{l}\text { Ferreira MVF; } \\
\text { Andrade D; } \\
\text { Ferreira } \text { AM }^{21}\end{array}$ & 2011 & $\begin{array}{l}\text { Revista da Escola } \\
\text { de Enfermagem } \\
\text { da USP/ BDENF }\end{array}$ & $\begin{array}{l}\text { Controle de infecção } \\
\text { relacionada a cateter } \\
\text { venoso central impregnado } \\
\text { com antissépticos: revisão } \\
\text { integrativa }\end{array}$ & $\begin{array}{l}\text { Demonstra a redução da colonização } \\
\text { microbiana através da utilização } \\
\text { de cateter venoso impregnado com } \\
\text { antissépticos. }\end{array}$ & 5 \\
\hline $\begin{array}{l}\text { Mendonça SHF; } \\
\text { Lacerda RA } \text { R2 }^{22}\end{array}$ & 2010 & $\begin{array}{l}\text { Acta Paulista de } \\
\text { Enfermagem/ } \\
\text { LILACS }\end{array}$ & $\begin{array}{l}\text { Impacto dos conectores } \\
\text { sem agulhas na infecção da } \\
\text { corrente sanguínea: revisão } \\
\text { sistemática }\end{array}$ & $\begin{array}{l}\text { A implantação de conectores } \\
\text { sem agulhas, quer valvulados ou } \\
\text { puncionáveis, com manutenção de } \\
\text { sistema fechado de infusão funcionam } \\
\text { como medida de prevenção com } \\
\text { impacto positivo na diminuição da } \\
\text { ocorrência de infecção da corrente } \\
\text { sanguínea. }\end{array}$ & 1 \\
\hline $\begin{array}{l}\text { Dallé J; Kuplich } \\
\text { NM; Santos RP; } \\
\text { Silveira DT } 23\end{array}$ & 2012 & $\begin{array}{c}\text { Revista do } \\
\text { Hospital das } \\
\text { Clínicas de Porto } \\
\text { Alegre (HCPA) / } \\
\text { LILACS }\end{array}$ & $\begin{array}{l}\text { Infecção relacionada a } \\
\text { cateter venoso central } \\
\text { após a implementação de } \\
\text { um conjunto de medidas } \\
\text { preventivas (bundle) no } \\
\text { centro de terapia intensiva do } \\
\text { Hospital de Clínicas de Porto } \\
\text { Alegre }\end{array}$ & $\begin{array}{l}\text { A implementação do bundle é } \\
\text { uma medida que gera melhorias } \\
\text { na estruturação dos processos } \\
\text { assistenciais. }\end{array}$ & 3 \\
\hline $\begin{array}{l}\text { Romanelli } \\
\text { RMC; Anchieta } \\
\text { LM; Mourão } \\
\text { MVA; Campos } \\
\text { FA; Loyola FC; } \\
\text { Mourão PHO }{ }^{25}\end{array}$ & 2013 & $\begin{array}{c}\text { Jornal de } \\
\text { Pediatria/ LILACS }\end{array}$ & $\begin{array}{l}\text { Fatores de risco e letalidade de } \\
\text { infecção da corrente sanguínea } \\
\text { laboratorialmente confirmada, } \\
\text { causada por patógenos não } \\
\text { contaminantes da pele em } \\
\text { recém-nascidos }\end{array}$ & $\begin{array}{l}\text { Descreve os fatores de risco para } \\
\text { recém-nascidos com relação a } \\
\text { infecção da corrente sanguínea } \\
\text { laboratorialmente confirmada. }\end{array}$ & 4 \\
\hline $\begin{array}{l}\text { Catarino CF; } \\
\text { Marins ACS; } \\
\text { Silva APAM; } \\
\text { Gomes AVO; } \\
\text { Nascimento } \\
\text { MAL }\end{array}$ & 2013 & $\begin{array}{l}\text { Revista de } \\
\text { pesquisa: } \\
\text { Cuidado é } \\
\text { Fundamental } \\
\text { online/ BDENF }\end{array}$ & $\begin{array}{l}\text { Perfil epidemiológico das } \\
\text { infecções primárias de corrente } \\
\text { sanguínea em uma unidade de } \\
\text { terapia intensiva neonatal }\end{array}$ & $\begin{array}{l}\text { Prevalência de microrganismos em } \\
\text { neonatos na abertura do quadro } \\
\text { infeccioso. }\end{array}$ & 6 \\
\hline
\end{tabular}

Fonte: dados da pesquisa.

de objetos entre pacientes pediátricos, a desnutrição aguda, a presença de anomalias congênitas, o uso de medicamentos como, antimicrobianos, nutrição parenteral total (NPT), corticosteroides, doenças hemato-oncológicas, crônico degenerativas e imunossupressoras ${ }^{19}$.

No Brasil, mesmo com programas e legislações vigentes para o controle da infecção hospitalar, percebese um aumento crescente de suas taxas, chegando a alcançar $15,5 \%$ dos pacientes internados. No cenário da UTI neonatal e pediátrica, foco deste estudo, essas taxas variam entre 18,9 a $57,7 \%{ }^{20}$.

Dito isto, a infecção hospitalar apresenta-se como um problema de saúde pública em todo o mundo que deve ser analisado sob uma perspectiva mais ampla, que abarque o desenvolvimento social, o entendimento sobre o processo saúde-doença e a adoção de modelos para a prestação de uma assistência segura e de qualidade. Nesta vertente, a reflexão sobre a infecção hospitalar deve voltar-se às medidas direcionadas ao modo como as políticas são introduzidas e distribuídas, à reformulação dos modelos tecnoassistenciais e avaliação das práticas assistenciais ${ }^{20}$.

Com o objetivo de garantir a qualidade e segurança da assistência prestada com foco na redução da incidência e da gravidade das IRAS, a Agência Nacional de Vigilância Sanitária (ANVISA) vem desenvolvendo diretrizes e normas, estabelecendo, em 2010, a meta nacional para reduzir em $30 \%$ a densidade de incidência de IPCS em pacientes portando um CVC ${ }^{14-21}$.

Essa determinação, fez com que os serviços de saúde reavaliassem suas práticas assistenciais, visando implementar estratégias para a redução de infecção de acordo com suas características a partir da adoção de práticas diferenciadas, como a utilização de cateter venoso impregnado com antisséptico demonstrando redução de colonização microbiana ${ }^{21}$, a implementação de conectores sem agulhas valvulados ou puncionáveis mantendo o sistema fechado de infusão ${ }^{22}$ e o seguimento de bundles específicios ${ }^{8-13-21-23-25}$.

De acordo com uma das evidências selecionadas ${ }^{17}, 90 \%$ das infecções associam-se ao CVC e que adoção de medidas 
básicas como higienização das mãos, precauções máximas de barreira, antissepsia com clorexidina, reavaliação diária da necessidade de manutenção do cateter, bem como adoção de um programa multidisciplinar representaram grande redução dos índices de infecção da corrente sanguínea, atendendo aos quesitos básicos preconizados pela National Healthcare Safety Network (NHSN).

Contudo, a implementação de tais medidas ainda se apresenta limitada frente à casuística da infecção hospitalar e frente ao reconhecimento das condições em que as práticas de assistência à saúde são realizadas pelos profissionais de saúde, bem como são classificadas diante das diretrizes e recomendações preconizadas ${ }^{7-8-26}$.

Frente a toda a problemática apontada, fica claro que a IPCS se apresenta com uma variedade de desfecho não só diagnóstico, mas como também prognóstica com consequências sistêmicas graves ao paciente, como a sepse por exemplo, geralmente manifestada de forma nefasta principalmente na população neonatal e pediátrica. Desta maneira, torna-se critério fundamental a adoção de boas práticas pelas instituições de saúde como forma de reduzir os índices de infecções relacionadas ao acesso vascular.

\section{Categoria 2- A sepse neonatal e pediátrica como principal complicação relacionada à IPCS}

Por seu turno, esta segunda categoria reúne evidências cientificas que relacionam a sepse como fator de risco para o desenvolvimento de IPCS.

Um estudo realizado sobre segurança do paciente revelou que a sepse se apresenta como um grave problema de saúde pública mundial e apontou que nos países em desenvolvimento esta questão torna-se ainda maior e ameaçadora, pois o risco relacionado aos cuidados de saúde pode ser até 20 vezes maior do que nos países considerados desenvolvidos ${ }^{27}$.

A incidência de sepse neonatal é altamente variável em diferentes hospitais. Uma comparação entre países revelou uma grande variação nas taxas de densidade de incidência, como pode ser observado nos Estados Unidos da América (EUA) de 3,6 a 18,1 infecções por 1.000 pacientes por dia ${ }^{28}$ e no Brasil de 25 infecções por 1.000 pacientes por dia ${ }^{29}$, o que requer ações severas de vigilância epidemiológica(30-31).

Vários fatores estão diretamente envolvidos na aquisição e desenvolvimento de sepse tardia na população neonatal e pediátrica, como baixo peso ao nascer, uso de dispositivos invasivos, atraso no início da nutrição enteral, nutrição parenteral total e complicações de prematuridade, como a persistência do canal arterial (PCA), displasia broncopulmonar e enterocolite necrosante ou outras situações que normalmente exigem breve intervenção cirúrgica ${ }^{28-29}$.

As variáveis peso e idade também foram evidenciadas por um estudo ${ }^{32}$ como fatores de risco para sepse neonatal, em que a densidade de incidência de IPCS variou entre 17,3 IPCS/1000 CVC por dia em recém-natos entre 1501 a
2500 gramas até 34,9 IPCS/1000 CVC por dia em recémnatos menores que 1000 gramas. Deste modo, a infecção é três vezes maior em neonatos e prematuros com baixo peso ao nascimento. Com relação a idade, 33,3\% eram RN a termos e 66,7\% pré-termos.

Assim, esta síntese de evidências mostra que dos recémnascidos acometidos pela IPCS, 61,5\% eram prematuros, o que acarreta um maior tempo de internação na UTI com consequente colonização por patógenos característico do ambiente hospitalar ${ }^{33-34}$.

Cerca de $1 \%$ dos neonatos nas unidades de terapia intensiva neonatal e 2 a 4,5\% dos recém-nascidos com baixo peso ao nascer apresentam septicemia causada por fungos, principalmente por Candida $\mathrm{spp}^{10-29-33-3}$ Os S. aureus apresentam-se como os agentes menos frequentes na infecção neonatal. Entretanto, a alta suscetibilidade dos neonatos e a alta virulência desses agentes coloca esta população em maior risco de morbidade e mortalidade, alcançando 55\% independente da resistência antimicrobiana ${ }^{29-33}$.

Considerando o exposto, compreende-se que a infecção primária de corrente sanguínea se destaca na população neonatal como uma grande causa de morbimortalidade, tornando-se imprescindível adoção de boas práticas que possam garantir a esta população menor risco ou complicação.

Desta maneira, ações simultâneas devem ser adotadas visando a redução da densidade de incidência de infecção, como o início precoce de alimentação enteral, amamentação, tempo de internação reduzido, além de treinamento e educação continuada para toda equipe ${ }^{28-34-35}$, bem como a implementação de protocolos como cirurgia segura, adoção de um conjunto de medidas para inserção, manutenção e retirada do CVC, além de programas educativos multidisciplinares, interdisciplinares e vigilância sobre os eventos de infecção por toda equipe envolvida na assistência prestada ${ }^{29-36-37}$.

Os achados demonstram que a ocorrência de infecção primária da corrente sanguínea relacionada ao acesso venoso vascular traz à população pediátrica e neonatal maiores agravos a saúde e condição clínica. Assim sendo, torna-se válida toda e qualquer iniciativa que privilegie uma ação nessa esfera como avaliar todas as nuances de interferência nas práxis assistenciais. Nessa leitura e compreensão, observa-se na atualidade grande avanço no atendimento à saúde com acesso a novas tecnologias, ferramentas diagnósticas, terapêuticas assim como a vigilância por meio da Comissão de Controle da Infecção Hospitalar (CCIH). De outra parte, temos os profissionais de saúde responsáveis pela compreensão e adoção constante destas na prestação de uma assistência segura e de qualidade assim como, de boas práticas que incluem atualização técnico-científica, implementação de protocolos, normas, rotinas, assim como o controle de indicadores nas unidades de saúde visando desta forma a redução da morbi-mortalidade proporcional associada à infecção hospitalar. 


\section{CONCLUSÃO}

Conclui-se que as evidências científicas mais recentes apontam que as IRAS figuram na atualidade como uma complicação frequente entre os pacientes hospitalizados, com destaque à IPCS, em que o CVC destacou-se como principal causa.

A IPCS se apresenta como um grave problema de saúde pública com forte impacto na morbimortalidade, sobretudo neonatal e pediátrica. 0 processo de trabalho em suas atuais configurações é insuficiente para avaliar e controlar a segurança e a qualidade da assistência prestada no contexto saúde, e que apesar das medidas de prevenção e controle da ICS estarem bem estabelecidas, as evidências apontam para a necessidade de mais investigações, pois, de acordo com algumas das próprias produções identificadas, pode haver desempenho insatisfatório entre os profissionais de saúde envolvidos em seu controle e prevenção.

Também foi possível observar a sepse como um dos fatores de risco para início do quadro de IPCS. Neste sentido, a conjugação entre atividades educativas e o exercício em revisitar a prática assistencial exercida, possibilitaria não só adoção de melhores práticas, mas uma análise crítica sobre os resultados destes processos, com vistas a prover uma assistência segura e de qualidade.

Apesar de o estudo ter alcançado o objetivo proposto, este teve como limitação o baixo nível de evidência entre os estudos identificados. Enquanto lacuna do conhecimento pode ser listada a necessidade de posteriores investigações de estudos de caso-controle ou randomizados que possam subsidiar o acompanhamento das medidas nacional e internacionalmente propostas, sobretudo se forem desenvolvidas em solo brasileiro. Há, portanto, a necessidade da continuidade ou do surgimento de novas discussões a respeito da efetiva adoção de práticas em saúde e em enfermagem que visem à qualidade da assistência para redução da morbimortalidade por IPCS na população pediátrica e neonatal.

\section{REFERÊNCIAS}

1. Lima AC. Complicações relacionadas a terapia intravenosa periférica em adultos cardiopatas internados [tese]. Campo Grande: Universidade Federal de Mato Grosso do Sul; [Internet]. 2013 [acesso em 10 jan 2016]. Disponível em: http://sistemas.ufms.br/sigpos/portal/trabalhos/ download/873/cursold:89.

2. Machado AF, Pedreira MLG, Chaud MN. Eventos adversos relacionados ao uso de cateteres intravenosos periféricos em crianças de acordo com tipos de curativos. Rev Latino-am Enfermagem. [Internet]. 2008 [acesso em 11 jan 2016]; 16(3):362-7. Disponível em: http://www. scielo.br/scielo.php?script=sci_arttext \&pid=S010411692008000300005 \&lng=en.

3. Belela ASC, Pedreira MLG, Peterlini MAS. Erros de Medicação em Pediatria. Rev Bras Enferm. [Internet]. 2011 [acesso em 14 jan 2016]; 64(3):563-9. Disponível em: http://www.scielo.br/ pdf/reben/v64n3/v64n3a22.pdf

4. Corrêa KLG, Almeida GMD, Almeida Júnior JN, Rossi F. Diferença de tempo de positividade: método útil no diagnóstico de infecção de corrente sanguínea relacionada com cateter?. J Bras Patol Med Lab. [Internet]. 2012 [acesso em 14 jan 2016]; 48(3):195-202. Disponível em: http://www. scielo.br/pdf/jbpml/v48n3/a07v48n3.pdf

5. Machado AF, Pedreira MLG, Chaud MN. Estudo prospectivo, randomizado e controlado sobre o tempo de permanência de cateteres venosos periféricos em crianças, segundo três tipos de curativos. Rev Latino-am Enfermagem. [Internet]. 2005 [acesso em 15 jan 2016]; 13(3): 291-8. Disponível em: http://www.scielo.br/pdf/rlae/v13n3/v13n3a02.pdf

6. Moncaio ACS, Figueiredo RM. Conhecimentos e práticas no uso do cateter periférico intermitente pela equipe de enfermagem. Rev Eletr Enf. [Internet]. 2009 [acesso em 18 jan 2016]; 11(3):620-7. Disponível em: http://www.fen.ufg. br/fen_revista/v11/n3/pdf/v11n3a20.pdf.

7. Vilela R, Dantas SRPE, Trabasso P. Equipe interdisciplinar reduz infecção sanguínea relacionada ao cateter venoso central em Unidade de Terapia Intensiva Pediátrica. Rev paul pediatr. [Internet].2010 [acesso em 22 jan 2016]; 28(4):292-98.Disponível em: http://www.scielo.br/pdf/rpp/v28n4/a02v28n4.pdf.

8. Jardim JM, Lacerda RA, Soares NJD, Nunes BK. Avaliação das práticas de prevenção e controle de infecção da corrente sanguínea em um hospital governamental. Rev Esc Enferm USP. [Internet]. 2013 [acesso em 21 jan 2016]; 47(1):3845. Disponível em: http://www.scielo.br/pdf/reeusp/v47n1/ a05v47n1.pdf.

9. Rosado V, Romanelli RMC, Camargos PAM. Fatores de risco e medidas preventivas para infecções da corrente sanguínea relacionadas ao cateter. J Pediatria. [Internet]. 2011 [acesso em 19 jan 2016]; 87(6):469-77. Disponível em: http://www. scielo.br/pdf/jped/v87n6/v87n06a03.pdf.

10. O'Grady NP, Alexander M, Burns LA, Dellinger EP, Garland J, Heard SO, et al. Guidelines for the preven on of intravascular catheter-related infections. J Vasc Interv Radiol. [Internet]. 2012 [acesso em 17 jan 2016]; 23(8): 997-1007. Disponível em: http://www.scielo.br/pdf/jped/v87n6/v87n06a03.pdf.

11. Santos El. Cuidado e prevenção das skin tears por enfermeiros: revisão integrativa de literatura. Rev Gaúcha Enferm. [Internet]. 2014 [acesso em 14 jan 2016]; 35(2): 142-9. Disponível em: http://seer.ufrgs.br/index.php/ RevistaGauchadeEnfermagem/article/view/45178.

12. Galvão CM, Sawada NO, Mendes IAC. A busca das melhores evidências. Rev Esc Enferm USP. [Internet]. 2003. [acesso em 23 jan 2016]; 37(4):43-50. Disponível em: http://www.scielo. br/pdf/reeusp/v37n4/05.pdf.

13. Institutefor Healthcare Improvement.GettingStartedKit;Prevent Central Line Infections How-to Guide. Cambridge: Institute for Healthcare Improvement; [Internet]. 2012 [acesso em 23 jan 2016].Disponívelem:http://www.ihi.org/resources/Pages/Tools/ GuidePreventCentralLineAssociatedBloodstreamlnfection.aspx.

14. Calil K, Silvino ZR, Valente GSC. Bundle para manuseio do cateter venoso central: pesquisa exploratória e descritiva. Online braz j nurs. [Internet]. 2013 [acesso em 21 jan 2016]; 12:713. Disponível em: http://www.objnursing.uff.br/index. php/nursing/article/view/4499. 
15. Worthington T, Elliott TSJ. Diagnosis of central venous catheter related infection in adult patient. J Infect. [Internet]. 2005 [acesso em 24 jan 2016]; 51(4):267-80. Disponível em: http:// www.journalofinfection.com/article/S0163-4453(05)001738/abstract.

16. Jardim JM. Avaliação das práticas de prevenção e controle da infecção da corrente sanguínea associada ao cateter venoso central de curta permanência por meio de indicadores clínicos. [tese]. São Paulo: Universidade de São Paulo; [Internet]. 2011 [acesso em 21 jan 2016]; Disponível em: http://www.teses.usp.br/teses/disponiveis/7/7139/tde21062011-151632/en.php.

17. Prates DB, Vieira MFM, Leite TS, Couto BRGM, Silva EU. Impacto de programa multidisciplinar para redução das densidades de incidência de infecção associada à assistência na UTI de hospital terciário em Belo Horizonte. Rev méd Minas Gerais. [Internet]. 2014 [acesso em 12 jan 2016]; 24(supl.6). Disponível em: http://rmmg.org/exportarpdf/1639/v24s6a12.pdf.

18. Mesiano ERAB, Hamann EM. Infecções da corrente sanguínea em pacientes em uso de cateter venoso central em unidades de terapia intensiva. Rev Latino-Am Enfermagem. [Internet]. 2007 [acesso em 14 jan 2016]; 15(3):453-9. Disponível em: http://www.scielo.br/pdf/rlae/v15n3/pt_v15n3a14.pdf.

19. Centers For Disease Control. Bloodstream Infection Event (Central Line-Associated Bloodstream Infection and Noncentral line-associated Bloodstream Infection). [internet]. [publicado em 2014 jan; acesso em 2015 out. 3]. Disponível em: http://www.cdc.gov/nhsn/PDFs/pscManual/4PSC_ CLABScurrent.pdf.

20. Sousa JO, Linhares TRC, Sousa DM, Soares EO. Infecção hospitalar em unidade de terapia intensiva neonatal: uma revisão da literatura. Rev. Interd. UNINOVAFAPI. Teresina. [Internet]. 2012 [acesso em 23 jan 2016]; 5(3): 77-80. Disponível em: http://www.uninovafapi.edu.br/sistemas/ revistainterdisciplinar/v5n3/rev/rev7_v5n3.pdf.

21. Agência Nacional de Vigilância Sanitária. Unidade de Investigação e Prevenção das Infecções e dos Eventos Adversos. Corrente Sanguínea: Critérios Nacionais de Infecções Relacionadas à Assistência à Saúde. [Internet]. 2009 [acesso em 10 jan 2016]; Disponível em: http://www. anvisa.gov.br/servicosaude/manuais/correntesanguinea.pdf.

22. Ferreira MVF, Andrade D, Ferreira AM. Controle de infecção relacionada a cateter venoso central impregnado com antissépticos: revisão integrativa. Rev Esc Enferm USP. [Internet]. 2011[acesso em 12 jan 2016]; 45(4): 1002-6. Disponível em: http://www.scielo.br/pdf/reeusp/v45n4/v45n4a30.pdf.

23. Mendonça SHF, Lacerda RA. Impacto dos conectores sem agulhas na infecção da corrente sanguínea: revisão sistemática. Acta Paul Enferm. [Internet]. 2010 [acesso em 12 jan 2016]; 23(4): 568-573. Disponível em: http://www.scielo. br/pdf/ape/v23n4/20.pdf.

24. Dallé Jessica, Kuplich Nádia Mora, Santos, Rodrigo Pires dos, Silveira Denise Tolfo. Infecção relacionada a cateter venoso central após a implementação de um conjunto de medidas preventivas (bundle) em centro de terapia Intensiva. Rev HCPA \& Fac Med Univ Fed Rio Gd do Sul. [Internet]. 2012 [acesso em 14 jan 2016]; 32(1): 10-17. Disponível em: http:// seer.ufrgs.br/hcpa/article/download/25148/16456.
25. Marschall J, Mermel LA, Classen D, Arias KM, Podgorny $\mathrm{K}$, Anderson DJ, et al. Strategies to prevent central lineassociated bloodstream infections in acute care hospitals. Infect Control Hosp Epidemiol. [Internet]. 2008 [acesso em 16 jan 2016]; 35(7): 753-71. Disponível em: http://www.jstor. org/stable/10.1086/676533.

26. Mckibben L, Horan TC, Tokars JI, Fowler G, Cardo DM, Pearson $M L$, et al. Guidance on public reporting of healthcare associated infections: recommendations of the Healthcare Infection Control Practices Advisory Committee. Infect Control Hosp Epidemiol. [Internet]. 2005 [acesso em 21 jan 2016]; 26(6): 580-7. Disponível em: https://www.cdc.gov/ hicpac/pdf/publicreportingguide.pdf.

27. Pessoa-Silva CL, Richtmann R, Calil R, Santos RM, Costa ML, Frota AC, Wey SB. Healthcare-associated infections among neonates in Brazil. Infect Control Hosp Epidemiol. [Internet]. 2004 [acesso em 21 jan 2016]; 25(9): 772-7. Disponível em: http://www.jstor.org/stable/10.1086/502475?seq=1\#page_ scan_tab_contents.

28. Paim R, Lorenzini E. Estratégias para prevenção da resistência bacteriana. Rev Cuid. [Internet]. 2014 [acesso em 16 jan 2016]; 5(2): 757-64. Disponível em: http://www.scielo.org. co/pdf/cuid/v5n2/v5n2a07.pdf.

29. Romanelli RMC, Anchieta LM, Mourão MVA, Campos FA, Loyola FC, Murão PHO, et al. Fatores de risco e letalidade de infecção da corrente sanguínea laboratorialmente confirmada, causada por patógenos não contaminantes da pele em recém-nascidos. J Pediatr. [Internet]. 2013 [acesso em 14 jan 2016]; 89(2): 189-196. Disponível em: http://www. scielo.br/pdf/jped/v89n2/v89n2a13.pdf.

30. Brasil. Critérios nacionais de infecções relacionadas à assistência à saúde [Internet]. Brasília, DF: Ministério da Saúde, Agência Nacional de Vigilância Sanitária, Neonatologia; 2010 [Acesso em 24 fev 2016]. Disponível em http://portal.anvisa.gov.br/wps/wcm/ connect/aa863580492e0b81b23ab314d16287af/ manual_neonatologia-\%2B03-09-2010-\%2Bnovo. pdf?MOD=AJPERES.

31. Landre-Peigne C, Ka AS, Peigne V, Bougere J, Seye MN, Imbert P. Efficacy of an infection control programme in reducing nosocomial bloodstream infections in a Senegalese neonatal unit. J Hosp Infect. [Internet]. 2011 [acesso em 24 fev 2016]; (79):161-5. Disponível em: http://www.journalofhospitalinfection.com/article/S01956701(11)00180-0/fulltext.

32. Stoll BJ, Hansen N, Fanaroff AA, Wright LL, Carlo WA, Ehrenkranz RA, et al. Late-onset sepsis in very low birth weight neonates: the experience of the NICHD Neonatal Research Network. Pediatrics. [Internet]. 2002 [acesso em 19 fev 2016]; 110(2): 285-91. Disponível em: http://pediatrics. aappublications.org/content/110/2/285.

33. Kawagoe JY, Segre CA, Pereira CR, Cardoso MF, Silva CV, Fukushima JT. Risk factors for nosocomial infections in critically ill newborns: a 5-year prospective cohort study. Am J Infect Control. [Internet]. 2001 [acesso em $24 \mathrm{fev}$ 2016]; 29(2):109-14. Disponível em: http://www.ajicjournal.org/ article/S0196-6553(01)80884-1/fulltext.

34. Catarino CF, Marins ACS, Silva APAM, Gomes AVO, Nascimento MAL. Perfil Epidemiológico das Infecções Primárias de 
Corrente Sanguínea em uma Unidade de Terapia Intensiva Neonatal. R pesq cuid fundam online. [Internet]. 2013 [acesso em 15 jan 2016]; 5(1):3229-37. Disponível em: http:// www.seer.unirio.br/index.php/cuidadofundamental/article/ view/2013/pdf_679.

35. Khoory BJ, Vino L, Dall'Agnola A, Fanos V. Candida infections in newborns: a review.J Chemother. [Internet]. 1999 [acesso em 21 fev 2016]; 11(5): 367-78. Disponível em: http://www.tandfonline. com/doi/pdf/10.1179/joc.1999.11.5.367?needAccess=true.

36. .Kayange N, Kamugisha E, Mwizamholya DL, Jeremiah S, Mshana SE. Predictors of positive blood culture and deaths among neonates with suspected neonatal sepsis in a tertiary hospital, Mwanza-Tanzania. BMC Pediatr. [Internet]. 2010 [acesso em 21 fev 2016]; 10:39. Disponível em: http://www. ncbi.nlm.nih.gov/pmc/articles/PMC2889942/pdf/14712431-10-39.pdf.

37. Srivastava S, Shetty N. Healthcare-associated infections in neonatal units: lessons from contrasting worlds. J Hosp Infect. [Internet]. 2007 [acesso em 21 fev 2016]; 65(4):292306. Disponível em: http://www.journalofhospitalinfection. com/article/S0195-6701(07)00040-0/fulltext. 